\title{
Indução à anestesia e recuperação de Betta splendens (Regan, 1910): uma proposta de utilização de um anestésico de baixo custo para uma espécie de peixe ornamental de grande apelo comercial
}

\author{
Daniela Sanches Ilgenfritz \\ Instituto Federal de Educação, Ciência e Tecnologia do Rio Grande do Sul (IFRS) \\ Campus Porto Alegre \\ (dani.fritz79@gmail.com)
}

Pâmella Chavez Ortiz

Instituto Federal de Educação, Ciência e Tecnologia do Rio Grande do Sul (IFRS)

Campus Porto Alegre

(pamellac.ortiz@gmail.com)

Caroline Pavin Lacerda

Instituto Federal de Educação, Ciência e Tecnologia do Rio Grande do Sul (IFRS)

Campus Porto Alegre

(carolpavin@msn.com)

Ângelo Cássio Magalhães Horn

Instituto Federal de Educação, Ciência e Tecnologia do Rio Grande do Sul (IFRS)

Campus Porto Alegre

(angelo.horn@poa.ifrs.edu.br)

Resumo: A manipulação sofrida por peixes destinados à comercialização gera um estado de estresse que favorece a contração de doenças e/ou a morte. Uma alternativa é a sedação/anestesia. Neste contexto, o óleo de cravo surge como uma alternativa promissora, pois induz a anestesia e propicia uma rápida recuperação. O objetivo do presente trabalho foi propor a concentração de óleo de cravo que melhor se adequa à indução da anestesia em Betta splendens e a sua recuperação. Para isto exemplares de $B$. splendens foram submetidos a soluções de óleo de cravo nas concentrações de $10,25,50,75,100$ ou 150mg/L ou a uma solução de álcool etílico com concentração de $1.075 \mathrm{mg} / \mathrm{L}$. Os resultados demostraram não haver indução à anestesia quando utilizada a concentração de $10 \mathrm{mg} / \mathrm{L}$ ou a exposição dos espécimes ao álcool etílico. Para outras concentrações de óleo de cravo percebeu-se uma redução na latência da anestesia com o aumento da concentração. O tempo de recuperação mostrou aumento quando concentrações de $25 \mathrm{mg} / \mathrm{L}$ e $50 \mathrm{mg} / \mathrm{L}$ foram comparadas. Conclui-se que a concentração de $25 \mathrm{mg} / \mathrm{L}$ é a melhor escolha para a anestesia de $B$. splendens em razão de sua latência e do maior tempo para que o último estágio de anestesia seja alcançado. 
Palavras-chave: Betta splendens; Anestesia; Óleo de cravo.

Induction to anesthesia and recovery of Betta splendens (Regan, 1910): a proposal for the use of a low-cost anesthetics for an ornamental fish species of great commercial appeal

\begin{abstract}
The manipulation suffered by commercial fish generates a state of stress that favors the contraction of diseases and/or death. An alternative is sedation/anesthesia. In this context, clove oil appears as a promising alternative, as it induces anesthesia and provides a rapid recovery. The aim of the present study was to propose the concentration of clove oil that is best suited to the induction of anesthesia in Betta splendens and its recovery. For this, $B$. splendens samples were submitted to solutions of clove oil at the concentrations of $10,25,50,75,100$ or $150 \mathrm{mg} / \mathrm{L}$ or to a solution of ethyl alcohol with concentration of $1.075 \mathrm{mg} / \mathrm{L}$. The results showed no induction to anesthesia when the concentration of $10 \mathrm{mg} / \mathrm{L}$ is used or the exposure of the specimens to ethyl alcohol occurs. For other concentrations of clove oil, a decrease in the latency of the anesthesia was observed with increasing concentrations. The recovery time showed an increase when concentrations of $25 \mathrm{mg} / \mathrm{L}$ and $50 \mathrm{mg} / \mathrm{L}$ were compared between them. It is concluded that the concentration of $25 \mathrm{mg} / \mathrm{L}$ is the best choice for the anesthesia of $B$. splendens because of its latency and the longer time for the last stage of anesthesia to be reached.
\end{abstract}

Keywords: Betta splendens; Anesthesia; Clove oil.

\title{
INTRODUÇÃO
}

A manipulação, invariavelmente, submete os animais que não estão habituados com esta prática a um estado de estresse. Para os peixes destinados a ornamentação ou ao consumo esta é uma condição extremamente prejudicial, uma vez que os conduz a aquisição de doenças ou a morte.

Uma alternativa visando reduzir o estresse causado pela manipulação é a sedação ou a anestesia. Há uma série de substâncias que atuam com esta finalidade, sendo a mais utilizada para peixes, por recomendação de diferentes órgãos que tratam de questões éticas referentes ao uso de animais nas mais diferentes áreas como o Conselho Federal de Medicina Veterinária do Brasil e a Associação Americana de Medicina Veterinária, os barbitúricos e seus derivados, (CFMV, 2002; AVMA, 2007). No Brasil, o anestésico mais utilizado com este fim é a benzocaína (ethyl-p-aminobenzoato) (GOMES et al., 2001). Apesar de ser um anestésico eficiente, apresentando uma série de aspectos favoráveis (NEIFFER e 
STAMPER, 2009), não é fácil de ser obtido, além de não possuir o melhor custobenefício quando comparado a outras alternativas (SOUZA, et al., 2012).

É consenso que o anestésico ideal deve produzir uma rápida indução à anestesia e à recuperação, gerando o mínimo de estresse; deve resultar em imobilização por tempo suficiente para o procedimento desejado e deve ter grande margem de segurança para o animal, para quem o manipula e para o ambiente (GOODMAN, 2012). Neste contexto, o óleo de cravo da índia vem sendo cada vez mais utilizado. Além de atender aos critérios citados acima, contudo, com um tempo de recuperação um pouco mais longo quando comparado a outros anestésicos, é de fácil obtenção e possui um excelente custo-benefício (NEIFFER e STAMPER, 2009).

O oléo de cravo é um produto natural extraído do cravo da índia (Syzygium aromaticum; sinônimo Cariophyllus aromaticus), sendo utilizado por seres humanos como anestésico tópico para dores de dente, de cabeça e nas articulações, desde a antiguidade (ROSS e ROSS, 2008). O efeito anestésico do óleo de cravo deve-se ao eugenol (4-allyl-2-methoxyphenol), isoeugenol e metileugenol, mas principalmente ao primeiro, que corresponde a aproximadamente $84 \%$ do conteúdo da substância vendida comercialmente (NEIFFER e STAMPER, 2009; JAVAHERY et al., 2012).

Muitos são os trabalhos experimentais que mais recentemente descreveram e/ou mensuraram os efeitos do óleo de cravo ou do eugenol como anestésico para diferentes espécies de peixes (HISANO et al., 2008; PEREIRA-DA-SILVA et al. 2009; SIMÕES et al., 2010; DELBON e RANZANI PAIVA, 2012; DIMER et al., 2012; ROTILI et al. 2012), com excelentes resultados, quanto a indução da anestesia e manutenção dos valores normais das variáveis fisiológicas, como os níveis de hormônios plasmáticos, a quantidade de células sanguíneas e a glicemia, dos animais testados (HOLLOWAY et al., 2004; VELISEK et al., 2006; MOREIRA et al., 2011). Seus efeitos como anestésico acabaram, inclusive, superando os efeitos obtidos por outras substâncias como a tricaína metano sulfonato (TMS ou MS-222), um derivado da benzocaína, amplamente utilizado e recomendado como anestésico na pesquisa com peixes, e a própria benzocaína (GRUSH et. al., 2004; HOLLOWAY et al., 2004; OLIVEIRA et al., 2009, SOUZA et. al. 2012).

Apesar de eficiente, de fácil obtenção e reduzido valor frente a outros anestésicos, a dosagem a ser utilizada para se obter o efeito anestésico varia com a espécie, a massa e a fase de desenvolvimento do peixe, assim como, o valor das 
variáveis ambientais e a temperatura (BORSKI e HODSON, 2003; NEIFFER e STAMPER, 2009; GOODMAN, 2012; JAVAHERY et al., 2012).

Betta splendens (Regan 1910) (Actinopterygii, Anabantoidei), também chamado de "a joia do oriente" ou "Betta esplêndido" por alguns criadores, é um peixe da subordem anabantoidei (ITIS, 2006), oriundo de diversos países da Ásia, como a Tailândia, a Indonésia, o Vietnã e a China, e que apresenta um comportamento tipicamente territorialista, demonstrando grande agressividade frente a um potencial invasor/competidor da mesma espécie (FARIA et al., 2006; KARINO e SOMEYA, 2007; VERBEEK et al., 2007). Em razão de constituírem-se em peixes ornamentais de fácil manutenção, possuem grande apelo comercial e, em decorrência disso, um número significativo de informações referentes a aspectos de sua biologia e criação (BORUCHOWITZ, 2006; FARIA et al., 2006; GOLDSTEIN, 2012; TULLOCK, 2006). Por outro lado, estudos propositivos tentando reduzir o estresse (e também, a morte, direta ou indiretamente associada ao estresse) causado pela manipulação, com fins comerciais ou científicos, são limitados para esta espécie (SOMMANI et al., 1999; PATTANASIRI et al., 2008; PATTANASIRI et al., 2016).

O objetivo deste trabalho foi propor a concentração de óleo de cravo que melhor se adequa à indução da anestesia e ao tempo de recuperação em exemplares adultos de $B$. splendens, visando reduzir o estresse dos animais durante a sua manipulação.

\section{MATERIAIS E MÉTODOS}

\section{Animais}

Trinta e seis espécimes adultos de $B$. splendens, de ambos os sexos, foram obtidos em um comércio local e levados ao Laboratório de Histologia do Instituto Federal de Educação, Ciência e Tecnologia do Rio Grande do Sul (IFRS) - Campus Porto Alegre, onde foram mantidos por uma semana em recipientes individuais, nos quais não era permitido que mantivessem contato visual com outros animais, para aclimatação. Os recipientes possuíam $0,5 \mathrm{~L}$ de água, com salinidade de 0,3\%. Todos os indivíduos foram submetidos à iluminação natural e temperatura em torno dos $28^{\circ} \mathrm{C}$, sendo alimentados, duas vezes ao dia com ração comercial. Duas vezes 
durante a semana foram medidos o pH e os níveis de amônia e nitritos da água, sendo esta parcialmente ( $25 \%$ do volume total) substituída.

Todos os procedimentos adotados nesta pesquisa com os espécimes respeitaram a Lei de Procedimentos para o Uso Científico de Animais (BRASIL, 2008), de 8 de outubro de 2008, regulamentada pelo Decreto $\mathrm{n}^{\circ} 6.899$, de 15 de julho de 2009 (BRASIL, 2009) e a Resolução 714, de 20 de junho de 2002, do Conselho Federal de Medicina Veterinária, que dispõe sobre procedimentos e métodos de eutanásia em animais (CFMV, 2002)

Este trabalho foi aprovado pelo Comité de Ética da Universidade Federal do Rio Grande do Sul (UFRGS), Brasil (Protocolo $\left.n^{\circ} 22625\right)$.

\section{Desenho experimental}

Os animais foram divididos em sete grupos de 3 a 4 animais e, após jejum de 40 horas, colocados em contato com uma solução de óleo de cravo nas concentrações de 5, 10, 25, 50, 100 e 150mg/L, assim como com uma solução de álcool etílico na concentração de $1.075 \mathrm{mg} / \mathrm{L}$ (que corresponde a maior concentração de álcool etílico encontrada entre as soluções de óleo de cravo).

Foram utilizados dois aquários de igual tamanho (200mm comprimento $X$ $90 \mathrm{~mm}$ profundidade $X 150 \mathrm{~mm}$ de altura) e com igual volume de água (1,5L). A água utilizada em ambos possuía as mesmas temperatura e salinidade utilizadas nos recipientes destinados à aclimatação. No primeiro aquário (aquário para anestesia, Figura 1) eram misturadas à água uma solução de óleo de cravo previamente solubilizada em etanol $95 \%$ (solução estoque) ou apenas etanol, até que se atingissem as concentrações descritas anteriormente. No segundo, apenas água (aquário para recuperação). Cada indivíduo, então, era introduzido no primeiro aquário, depois de intensa oxigenação da água por 5 minutos, e o tempo de indução à anestesia (em minutos), cronometrado e anotado. Após, os animais eram transferidos para o segundo aquário, iniciando a contagem do tempo de recuperação (em min). 
Figura 1 - Aquário para anestesia.

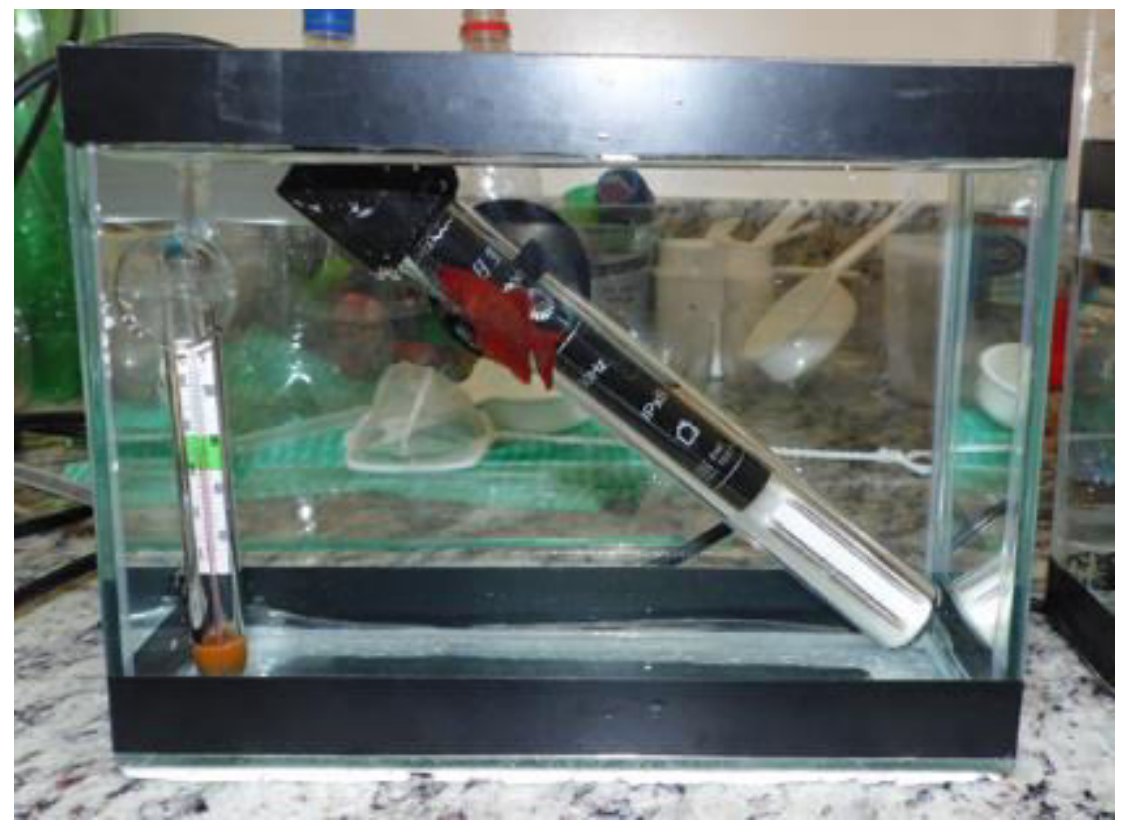

Fonte: os autores.

O tempo de permanência no aquário para anestesia se estendia até o animal atingir o estágio 4 de anestesia (Quadro 1) ou ter completado uma hora no mesmo. Ao contrário da anestesia, não foi estabelecido um tempo máximo para a permanência no aquário para a recuperação.

Após recuperados, os animais tiveram o seu comprimento padrão e massa aferidos, utilizando-se um paquímetro analógico série 125 (Starrett, Brasil), em milímetros, e uma balança semianalítica BK300 (Gehaka, Brasil), em gramas, respectivamente.

Passada a manipulação experimental os animais foram observados por uma semana e a fim de registrar possíveis mortes.

\section{Anestésico}

O óleo de cravo extraído de Cariophyllus aromaticus (densidade de 1,04 g/ml) foi obtido em farmácia de manipulação e diluído em etanol 95\% a 10\%, para facilitar sua solubilização em água (solução estoque). Esta solução estoque foi, então, diluída na água do aquário para anestesia até obter-se as concentrações desejadas para os grupos de animais utilizados, sendo intensamente oxigenada por 5 minutos antes da introdução do animal em seu interior, como descrito anteriormente. 


\section{Estágios de anestesia e recuperação}

Foram identificados para os peixes submetidos ao óleo de cravo, pelas características comportamentais, quatro estágios de anestesia e mais a recuperação. O tempo (em minutos) que cada animal levou para atingir cada um dos estágios de anestesia foi anotado e uma tabela representando a relação entre a concentração do anestésico, o estágio de anestesia e este tempo, construída (Tabela 1). Da mesma forma o tempo (em minutos) necessário para que cada animal se recuperasse da anestesia foi registrado e expresso nesta mesma tabela (Tabela 1).

Os estágios de anestesia e a recuperação utilizados foram aqueles descritos por WOODY et al., (2002) e caracterizados no Quadro 1.

Quadro 1 - Características comportamentais para peixes durante diferentes estágios de anestesia e recuperação.

\begin{tabular}{|c|l|}
\hline Estágio de anestesia & \multicolumn{1}{|c|}{ Características comportamentais } \\
\hline 1 & Movimentos operculares lentos ou erráticos \\
\hline 2 & $\begin{array}{l}\text { Dificuldade de manter a posição normal de nado enquanto parado e } \\
\text { perda esporádica do equilíbrio. }\end{array}$ \\
\hline 3 & $\begin{array}{l}\text { Perda completa do equilíbrio com incapacidade de assumir a posição } \\
\text { normal de nado. }\end{array}$ \\
\hline 4 & $\begin{array}{l}\text { Sem reação à manipulação ou a um forte estímulo no pedúnculo } \\
\text { caudal. }\end{array}$ \\
\hline Recuperação & $\begin{array}{l}\text { Recuperação da posição normal de nado e da capacidade de nadar } \\
\text { horizontalmente. }\end{array}$ \\
\hline
\end{tabular}

Fonte: WOODY et al. (2002).

\section{RESULTADOS}

Nossos resultados demonstraram que os exemplares de B. splendens, de ambos os sexos, com comprimento padrão entre $27,2 \mathrm{~mm}$ e $44,8 \mathrm{~mm}$ e massa variando de $0,61 \mathrm{~g}$ a 2,29g, apresentaram uma latência menor para ingressar nos estágios de anestesia com valores mais altos a medida que a concentração do anestésico ao qual foram expostos aumentou (Tabela 1). Por exemplo, animais expostos a concentrações de anestésico de 25mg/L atingiram o estágio 3 apenas depois de transcorridos 7 minutos do início da exposição ao óleo de cravo, enquanto 
animais submetidos a uma concentração de 100mg/L atingiram o mesmo estágio já no primeiro minuto de exposição (Tabela 1).

Não houve indução à anestesia quando se utilizou a concentração de $10 \mathrm{mg} / \mathrm{L}$ de óleo de cravo, mesmo depois de transcorrida uma hora da exposição ao anestésico (Tabela 1).

Por outro lado, o tempo de recuperação dos animais apresentou grande variação entre as diferentes concentrações e entre os diferentes animais submetidos a uma mesma concentração do anestésico (Tabela 1). Cabe atentar que, como exemplo, ao comparar animais expostos à uma concentração de óleo de cravo de $25 \mathrm{mg} / \mathrm{L}$ com aqueles submetidos a uma concentração equivalente a $100 \mathrm{mg} / \mathrm{L}$, o tempo máximo necessário à recuperação dos representantes do segundo grupo é inferior ao tempo máximo de recuperação obtido pelos animais do primeiro grupo, não fazendo qualquer sentido a não ser sob a lógica da variabilidade.

O álcool etílico, em uma concentração equivalente a $1.075 \mathrm{mg} / \mathrm{L}$, valor igual à maior concentração desta substância misturada ao óleo de cravo (encontrada na solução de óleo de cravo de $150 \mathrm{mg} / \mathrm{L}$ ) e com o papel de aumentar a solubilidade do anestésico à água, não apresentou qualquer efeito anestésico.

Transcorrida uma semana da exposição à solução de óleo de cravo ou a de álcool etílico não houve morte de qualquer espécime testado. 

apelo comercial

Tabela 1 - Tempo para indução aos estágios de anestesia e para a recuperação em $B$. splendens expostos a soluções de óleo de cravo com diferentes concentrações.

\begin{tabular}{|c|c|c|c|c|c|c|c|c|c|c|c|c|c|c|c|c|c|}
\hline \multirow{3}{*}{$\begin{array}{c}\text { Concentração } \\
(\mathrm{mg} / \mathrm{L})\end{array}$} & \multicolumn{16}{|c|}{ Anestesia (min) } & \multirow{3}{*}{$\begin{array}{c}\text { Recuperação } \\
\text { (min) }\end{array}$} \\
\hline & \multicolumn{10}{|c|}{10} & \multicolumn{2}{|l|}{20} & \multirow[t]{2}{*}{30} & \multirow[t]{2}{*}{40} & \multirow[t]{2}{*}{50} & \multirow[t]{2}{*}{60} & \\
\hline & 1 & 2 & 3 & 4 & 5 & 6 & 7 & 8 & 9 & 10 & 15 & 20 & & & & & \\
\hline 10 & 0 & 0 & 0 & 0 & 0 & 0 & 0 & 0 & 0 & 0 & 0 & 0 & 0 & 0 & 0 & 0 & $\mathrm{~N}$ \\
\hline 25 & 0,1 & 0,1 & 0,1 & 0,1 & 0,1 & 0,1 & $1,2,3$ & $1,2,3$ & $1,2,3$ & $1,2,3$ & $1,2,3$ & 2,3 & 2,4 & 2 & 2,3 & 2,3 & $2-8$ \\
\hline 50 & 1 & 1,2 & $1,2,3$ & 2,3 & 2,3 & 3 & 3 & 3 & 3 & 3 & 3,4 & 3,4 & 4 & - & - & - & $9-12$ \\
\hline 75 & 1,2 & 2,3 & 2,3 & 3 & 3,4 & 3 & 3 & 3 & 3,4 & 3 & 4 & - & - & - & - & - & $5-13$ \\
\hline 100 & $1,2,3$ & 3 & 3 & 3 & 3,4 & 3,4 & 4 & - & - & - & - & - & - & - & - & - & $5-7$ \\
\hline 150 & $1,2,3$ & 3 & 3,4 & 3,4 & 3 & 4 & - & - & - & - & - & - & - & - & - & - & $5-10$ \\
\hline
\end{tabular}

0, Ausência de anestesia; 1, Estágio 1; 2, Estágio 2; 3, Estágio 3 e 4, Estágio 4. N, Não houve necessidade de recuperação. 


\section{DISCUSSÃO}

Apesar do efeito anestésico do óleo de cravo já ter sido aferido em $B$. splendens (PATTANASIRI et al., 2008), tanto as concentrações utilizadas quanto os tempos de permanência dos animais em contato com a substância foram diferentes daqueles propostos em nosso trabalho. Enquanto, no trabalho de 2008 as concentrações variavam de 5 a 100ppm e o tempo de exposição máximo era de 48 horas em nosso trabalho a concentração máxima foi de $150 \mathrm{mg} / \mathrm{L}$ e o tempo máximo de 60min.

Diferente do trabalho de PATTANASIRI et al., (2008), concentrações iguais a $10 \mathrm{mg} / \mathrm{L}$, na presente pesquisa, não induziram à anestesia, nem mesmo após transcorrida uma hora de exposição. Por outro lado, todas as concentrações de óleo de cravo iguais ou superiores a $25 \mathrm{mg} / \mathrm{L}$ conduziram à anestesia os exemplares de $B$. splendens já no primeiro minuto dos testes. No trabalho de 2008, a uma concentração de $20 \mathrm{ppm}$, o óleo de cravo induziu a anestesia apenas após transcorridos 30min de exposição, ocorrendo o mesmo com uma concentração ligeiramente inferior (15ppm). Concentração capaz de induzir a anestesia depois de transcorridos 5 minutos de contato dos peixes com o anestésico foi observada apenas em concentrações superiores a 50ppm (PATTANASIRI et al., 2008). Tais discrepâncias observadas entre os dois trabalhos podem ser explicadas pela concentração de eugenol encontrada nas amostras de óleo de cravo utilizadas, uma vez que os autores citados acima obtiveram o óleo de Syzigium aromaticum.

Adicionalmente, a presente investigação mostrou que a latência para se atingir estágios de anestesia cada vez mais altos (do 1 ao 4) tornou-se cada vez menor a medida que havia um aumento na concentração do anestésico, concluindose que a ação do óleo de cravo na indução à anestesia, mostrou um padrão dosedependente. Tal dado concorda com aquele já expresso para $B$. splendens (PATTANASIRI et al., 2008) e para outras espécies de peixes que utilizaram o óleo de cravo como anestésico (WOODY et al., 2002; HISANO et al., 2008; NEIFFER e STAMPER, 2009; OLIVEIRA et al., 2009; PEREIRA-DA-SILVA et al., 2009; SIMÕES et al., 2010). Assim, utilizando-se a concentração de 25mg/L, os estágios 2 e 3 são obtidos após transcorridos 7 minutos de exposição, enquanto o estágio 4 só advêm aos 20 minutos de contato do animal com o anestésico. Para todas as demais concentrações os estágios 2 ou 3 são obtidos já nos primeiros 2 minutos de 
exposição, enquanto o estágio 4 é observado após 15minutos, para uma concentração de 50mg/L, 5 minutos, para concentrações de 75mg/L e 100mg/L e 3 minutos para a concentração de $150 \mathrm{mg} / \mathrm{L}$.

Desta forma, com base na latência observada, a concentração de óleo de cravo de $25 \mathrm{mg} / \mathrm{L}$ parece ser a que melhor se adequa a reduzir o estresse causado pela manipulação com certa margem de segurança, uma vez que exige um tempo para a indução à anestesia (estágio 1) reduzido (1 a 15min) e cerca de trinta minutos para que o estágio 4 seja alcançado, estágio este que poderia conduzir o animal à morte pela interrupção da ventilação e redução do débito cardíaco (AVMA, 2007).

Além da concentração indicada acima outras concentrações poderiam vir a ser testadas com a mesma finalidade, como concentrações intermediárias entre os $10 \mathrm{mg} / \mathrm{L}$ e $25 \mathrm{mg} / \mathrm{L}$.

Quanto ao tempo de recuperação, surpreendentemente, os dados não mostraram a existência de uma clara relação dose-dependente (quanto maior a concentração do anestésico maior o tempo de recuperação), como ocorre com outros peixes (PEREIRA-DA-SILVA et al., 2009; SIMÕES et al., 2010) e mesmo com B. splendens (PATTANASIRI et al., 2008). Apesar de se observar um padrão de dose-dependência quando comparadas as concentrações de $25 \mathrm{mg} / \mathrm{L}$ e $50 \mathrm{mg} / \mathrm{L}$, percebe-se, que o tempo máximo de recuperação de animais expostos a concentrações de $100 \mathrm{mg} / \mathrm{L}$ e $150 \mathrm{mg} / \mathrm{L}$ são menores do que daqueles exemplares submetidos a concentrações de $25 \mathrm{mg} / \mathrm{L}$ e $50 \mathrm{mg} / \mathrm{L}$, respectivamente. Esta discrepância parece ser resultado de fatores intrínsecos, próprios dos animais testados, e está calcada na variabilidade intraespecífica da população amostrada. Uma maneira de reduzir esta variabilidade seria aumentando o número de animais testados em cada uma das concentrações propostas.

Concluindo, este trabalho objetivou testar a ação de diferentes concentrações de óleo de cravo na indução da anestesia e no tempo de recuperação de exemplares adultos de $B$. splendens, com a finalidade de propor sua utilização na redução do estresse causado pela manipulação. Os dados apontam que a concentração de $25 \mathrm{mg} / \mathrm{L}$ de óleo de cravo é a que melhor se adequa ao objetivo proposto, em razão de sua latência reduzida e do longo tempo necessário para conduzir o animal ao estágio mais profundo de anestesia. 


\section{REFERÊNCIAS}

AVMA. American Veterinary Medical Association: Guidelines on Euthanasia. (Formerly Report of the AVMA Panel on Euthanasia). Disponível em:< http://www.avma.org/issues/animal_welfare/euthanasia.pdf>. Acesso em: 19 de setembro. 2007.

BORSKI, R.J., HODSON, R.G. Fish Research and the Institutional Animal Care and Use Commitee. ILAR Journal, 44(4): 286-294. 2003.

BORUCHOWITZ, D. E. Aquarium Care of Bettas. Neptune City: Tfh Pubns Inc. 2006.

BRASIL. Lei 11.794, de 8 de outubro de 2008. Procedimentos para o Uso Científico de Animais. In: Diário Oficial da União, Brasília, v. 145, n. 196. p.1. 2008.

BRASIL. Decreto 6.899, de 15 de julho de 2009. Dispõe sobre a composição do Conselho Nacional de Controle de Experimentação Animal - CONCEA, estabelece as normas para o seu funcionamento e de sua Secretaria-Executiva, cria o Cadastro das Instituições de Uso Científico de Animais - CIUCA, mediante a regulamentação da Lei no 11.794, de 8 de outubro de 2008, que dispõe sobre procedimentos para o uso científico de animais, e dá outras providências. In: Diário Oficial da União, Brasília, n. 134. p.2. 2009.

CFMV. Resolução n. 714, de 20 de julho de 2002. Dispõe sobre procedimentos e métodos de eutanásia e animais, e dá outras providências. In: Diário Oficial da União, Brasília, n. 118. p.201. 2002.

DELBON, M.C., RANZANI PAIVA, M. J. T. Eugenol em juvenis de tilápia do Nilo: concentrações e administrações sucessivas. Bol. Inst. Pesca, São Paulo, 38(1): 43-52. 2012.

DIEMER, O., NEU, D. H., BITTENCOURT, F., SIGNOR, A., BOSCOLO, W.R., FEIDEN, A. Eugenol como anestésico para jundiá (Rhamdia voulezi) em diferentes pesos. Semina: Ciências Agrárias, Londrina, 33(4): 1495-1500. 2012.

FARIA, P. M. C.; CREPALDI, D. V.; TEIXEIRA, E. A; RIBEIRO, L. P.; SOUZA, A. B.; CARVALHO, D. C.; MELO, D. C. SALIBA, E. O. S. Criação, manejo e reprodução do peixe Betta splendens (Regan, 1910). Rev. Bras. Reprod Anim. 30(3/4): 134-149, 2006.

GOLDSTEIN, R. J. Bettas: Barron's complete pet Owner's Manuals. 2 ed. New York: Barron's Educational Series. 2012.

GOMES, L.C.; CHIPPAR-GOMES, A.R.; LOPES, N.P.; ROUBACH, R.; ARAÚJOLIMA, C.A.R.M. Efficacy of benzocaine as an anesthetic in juvenile tambaqui Colossoma macropomum. Journal of the World Aquaculture Society, Baton Rouge, 32 (4): 426-431. 2001. 
GOODMAN, G. Guidelines for anaesthesia and analgesia of fish. Disponível em: < http://www.norecopa.no/norecopa/vedlegg/Guidelines-for-anaesthesia-andanalgesia-of-fish.pdf > . Acesso em 21 de setembro. 2012.

GRUSH, J., Noakes, D.L.G, Moccia, R.D. The Efficacy of Clove Oil as an anesthetic for the Zebrafish, Danio rerio (Hamilton), Zebrafish, 1(1):46-53. 2004.

HISANO, H., ISHIKAWA, M. M., FERREIRA, R. A., BULGARELLI, A. L. A., COSTA, T. R., PÁDUA, S. B. Tempo de indução e de recuperação de dourados Salminus brasiliensis (Cuvier, 1816), submetidos a diferentes concenrações de óleo de cravo Eugenia sp. Acta Sci. Biol. Sci., 30(3): 303307. 2008.

HOLLOWAY, A. C., KEENE, J. L., NOAKES, D. G., MOCCIA, R.D. Effects of clove oil and MS-222 on blood hormone profiles in rainbow trout

Oncorhyncus mykiss, Walbaum. Aquaculture Research, 35: 1025-1030. 2004.

INTEGRATED TAXONOMIC INFORMATION SYSTEM (ITIS). Catalog of fishes. Disponível em http://www.itis.usda.gov. Acesso em 16 de março de 2011.

JAVAHERY, S., NEKOUBIN, H., MORADLU, A. H. Effect of anesthesia with clove oil in fish (Review). Fish Physiol. Biochem., 38: 1545-1552. 2012.

KARINO, K.; SOMEYA, C. The influence of sex, line, and fight experience on aggressiveness of the Siamese fighting fish in intrasexual competition. Behavioural Process. 75: 283-289, 2007.

MOREIRA, A.G.L., TEIXEIRA, E. G., MOREIRA, R. L., FARIAS, W. R. L. Glicose plasmática em juvenis de tilápia do Nilo anestesiados com óleo de cravo. Rev. Bras. Saúde Prod. An., Salvador, 12(3): 794-804. 2011.

NEIFFER, D. L., STAMPER, M. A. Fish Sedation, Anesthesia, Analgesia, and euthanasia: Considerations, Methods, and Types of Drugs. ILAR Journal, 50(4): 343-360. 2009.

OLIVEIRA, J. R., CARMO, J. L., OLIVEIRA, K. K. C., SOARES, M. C. F. Cloreto de sódio, bezocaína e óleo de carvo-da-índia na água de transporte de tilápia-do-nilo. R. Bras. Zootec., 38(7): 1163-1169. 2009.

PATTANASIRI T., TAPARHUDEE, W., SUPPAKUL P. Antianxiety activity of clove oil and its principal constituent, and possible application in active packaging for transportation of Siamese fighting fish. The Proceedings of 16th IAPRI World Conference on Packaging. June 8-12, 2008. Bangkok, Thailand.

PATTANASIRI T., TAPARHUDEE, W., SUPPAKUL P. Anaesthetic efficacy of clove oil-coated LDPE bag on improving water quality and survival in the Siamese fighting fish, Betta splendens, during transportation. Aquacult int, DOI: 10.1007/s10499-016-0022-0. 2016. 
PEREIRA-DA-SILVA, E. M., OLIVEIRA, R. H. F., RIBEIRO, M. A. R., COPPOLA, M. P. Efeito anestésico do óleo de cravo em alevinos de lambari. Ciência Rural, Santa Maria, 39(6): 1851-1856. 2009.

ROTILI, D. A., DEVENS, M. A., DIEMER, O., LORENZ, E. K., LAZZARI, R., BOSCOLO, W. R. Uso do eugenol como anestésico em pacu. Pesq. Agropec. Trop., Goiânia, 42(3): 288-294. 2012.

ROSS, L. G., ROSS, B. Anaesthetic \& Sedative Techniques for Aquatic Animals. $3^{\text {rd }}$ Ed. Oxford: Blackwell Plubishing. 2008. 221p.

SIMÕES, L. N., PAIVA, G., GOMES, L. C. Óleo de cravo como anestésico em adultos de tilápia-do-nilo. Pesq. Agropec. Bras., Brasília, 45(12): 1472-1477. 2010.

SOMMANI, A., KERDKRIENGKAI, S., INGKAPAIROJ, N. Effect of MS-222 and Benzocaine to the transportation of Siamese fighting fish (Betta splendens Regan). Kasetsart J. (Nat. Sci.), 33: 368-376. 1999.

SOUZA, R. A. R., CARVALHO, C.V.A., NUNES, F. F., SCOPEL, B. R., GUARIZI, J. D., TSUZUKI, M. Y. Efeito comparativo da benzocaína, mentol e eugenol como anestésicos para juvenis de Robalo peva. Bol. Inst. Pesca, São Paulo, 38(3): 247-255. 2012.

TULLOCK, J. H. Betta: Your Happy Healthy Pet. 2 ed. New York: Howell Book House. 2006.

VELISEK, J, WLASOW, T., GOMULKA, P., SVOBODOVA, Z. NOVOTNY, L, ZIOMEK, E. Effects of Clove Oil and Anaesthesia on European Catfish (Silurus glanis L.). Acta Vet. Brno, 75: 99-106. 2006.

VERBEEK, P.; IWAMOTO, T.; MURAKAMI, N. Differences in aggression between wild-type and domesticated figthing fish are context dependent. Anim. Behav.73: 75-83, 2007.

WOODY, C.A., NELSON, J., RAMSTAD, K. Clove oil as an anaesthetic for adult sockeye salmon: field trials. Journal of Fish Biology, 60: 340-347. 2002. 